\title{
Fratura do complexo zigomático-orbitário: uma abordagem cirúrgica
}

\author{
Orbitozygomatic fracture: a surgical approach
}

Fractura del complejo cigomático-orbitario: un enfoque quirúrgico Gaya Bertoldo CASSIANO ${ }^{1}$

Francielly Thomas FIGUEIREDO ${ }^{2}$

Gustavo Silva PELISSARO 3

Jose Carlos Garcia de MENDONÇA ${ }^{4}$

Julio Cesar Leite da SILVA ${ }^{5}$

Ellen Cristina GAETTI JARDIM ${ }^{5}$

${ }^{1}$ Graduanda da Faculdade de Odontologia da Universidade Federal de Mato Grosso do Sul (Faodo/UFMS)

${ }^{2}$ Cirurgiã-dentista Residente em Cirurgia e Traumatologia Bucomaxilofacial da Faodo/UFMS

${ }^{3}$ Doutorando da Faculdade de Medicina da UFMS e Preceptor da Residência em Cirurgia e Traumatologia Bucomaxilofacial da Faodo/UFMS

${ }^{4}$ Professor Doutor Coordenador da Residência em Cirurgia e Traumatologia Bucomaxilofacial da Faodo/UFMS

${ }^{5}$ Professor(a) Doutor(a) Tutor da Residência em Cirurgia e Traumatologia Bucomaxilofacial da Faodo/UFMS

\section{Resumo}

Em virtude da sua projeção, uma das regiões mais acometidas por injúrias é o complexo zigomático, muitos casos de fraturas, provenientes da ação direta de forças, fragilizam sua estrutura e resultam na perda de sua curvatura convexa normal. Sendo assim, objetivo desse trabalho é relatar a abordagem cirúrgica de um caso de traumatismo facial secundário à agressão física, atendido no Hospital Universitário Maria Aparecida Pedrossian (HUMAP). Paciente do sexo masculino, branco, vítima de trauma, buscou o Pronto Atendimento, com hemiface esquerda edemaciada, equimose e assimetria facial associadas a oclusão palpebral, hiposfagma. sendo que aos exames complementares de imagem foram evidenciadas fraturas dos ossos do complexo zigomático orbitário. Em virtude da complexidade das fraturas bem como a possibilidade de comprometimento ocular, fez-se necessário a associação de conhecimentos referentes a diversas especialidades tais como: Cirurgia Bucomaxilofacial, Cardiologia e Oftalmologia. Em virtude do grau de deslocamento das fraturas optou-se pelo procedimento cirúrgico de redução e fixação das mesmas sob anestesia geral utilizando os acessos cirúrgicos subtarsal, subciliar e intraoral. As fixações foram realizadas com placas e parafusos de titânio do sistema $1.5 \mathrm{~mm}$ para cada acesso efetuado, em material de titânio. O presente caso, devido sua extensão e envolvimento de assoalho orbitário necessitou de três pontos para sua adequada fixação e apesar da complexidade, o caso mostrou sucesso da terapêutica empregada.

Descritores: Traumatismos Faciais; Fraturas Zigomáticas; Fraturas Maxilares.

\section{Abstract}

Due to its projection, one of the most affected regions it is the zygomatic complex, which in many cases of fractures, because of the direct action of forces, weakens and result in loss of your normal convex curvature. Therefore, the goal of this paper is to report the surgical approach of a case: a secundary facial trauma due to fisical assault, attended in Maria Aparecida Pedrossian College Hospital (HUMAP). Male pacient, white, victim of trauma, search the Emergency Room with swollen left hemiface, ecchymosis and facial asymmetry with eyelid occlusion, hyposfagma, in which the complementary tests of image showed bone orbitozygomatic fracture. Because of the complex fractures, as well as occular complication possibility, it was necessary to associate knowledge of many specialties, such as: Oral and maxillofacial surgery, Cardiology and Ophthalmology. In virtue of the big displacement of fractures the option of treatment was surgical procedure of reduction and setting of the fractures under general anesthesia usying subtarsal, subciliar and intraoral surgical accesses. For which access done, the settings were made usying Titanium plates and screws of the system $1.5 \mathrm{~mm}$. In this case, because of its orbital floor extension and involvement, three points of proper fixation was necessary and, despite your complexity, the case showed success of therapy used.

Descriptors: Facial Injuries; Zygomatic Fractures; Maxillary Fractures.

\section{Resumen}

Por su proyección, una de las regiones más afectadas por las lesiones es el complejo cigomático, muchos casos de fracturas, resultantes de la acción directa de fuerzas, debilitan su estructura y resultan en la pérdida de su curvatura convexa normal. Por tanto, el objetivo de este trabajo es reportar el abordaje quirúrgico de un caso de trauma facial secundario a agresión física, atendido en el Hospital Universitário Maria Aparecida Pedrossian (HUMAP). Varón, paciente blanco, víctima de traumatismo, que acudió a urgencias, con hemicara izquierda inflamada, equimosis y asimetría facial asociada a oclusión palpebral, hiposfagma. los exámenes de imagen complementarios revelaron fracturas de los huesos del complejo cigomático orbitario. Debido a la complejidad de las fracturas así como a la posibilidad de afectación ocular, fue necesario asociar conocimientos relacionados con varias especialidades como: Cirugía Maxilofacial, Cardiología y Oftalmología. Debido al grado de desplazamiento de las fracturas, optamos por el procedimiento quirúrgico para reducirlas y repararlas bajo anestesia general mediante los abordajes quirúrgicos subtarsal, subciliar e intraoral. Las fijaciones se realizaron con placas y tornillos de titanio del sistema de 1,5 mm por cada acceso realizado, en material de titanio. El presente caso, por su extensión y afectación del suelo orbitario, requirió tres puntos para su adecuada fijación y, a pesar de la complejidad, el caso mostró éxito de la terapia empleada.

Descriptores: Traumatismos Faciales; Fracturas Cigomáticas; Fracturas Maxilares.

INTRODUÇÃO

$$
\text { O osso zigomático define a projeção }
$$

antero-lateral da face e se articula com os ossos frontal, esfenoidal, temporal e maxilar sendo responsável pela proteção do conteúdo orbital e do contorno médio-facial ${ }^{1,2}$. A fratura do complexo zigomático é uma das lesões faciais mais comuns no trauma maxilofacial e aparece predominantemente em homens jovens adultos ${ }^{2,3}$.

$$
\text { A etiologia das fraturas do complexo }
$$

zigomático-orbitário (CZO) inclui principalmente acidentes de trânsito, agressões físicas violentas, quedas e lesões esportivas com variação geográfica e sociodemográfica na epidemiologia das fraturas maxilofaciais devido a fatores socioeconômicos, culturais e ambientais $^{3}$.

O sexo masculino é o mais acometido, representando $86,6 \%$ das fraturas de face, quando comparado ao sexo feminino $(13,4 \%)$, 
estabelecendo uma proporção de aproximadamente 6:1. Quanto aos agentes etiológicos, foram registrados $38,8 \%$ de casos de agressão; $14,2 \%$ de casos de acidente automobilístico; $13,4 \%$ de casos de acidente motociclístico; $9 \%$ de casos de queda; $6,7 \%$ de casos de atropelamento; $5,2 \%$ de casos de acidente esportivo; $5,2 \%$ de casos de acidente de trabalho; $4,5 \%$ de casos de ferimento por arma de fogo e $3 \%$ de casos de acidente ciclístico ${ }^{4}$.

Sendo assim, o cirurgião bucomaxilofacial deve procurar obter a história do trauma (tipo, intensidade, tempo decorrido), realizar o exame físico e solicitar exames de imagem. Os principais sinais e sintomas incluem aprisionamento do músculo extraocular, dormência no território de inervação do nervo infraorbitário, epistaxe, assimetria facial com afundamento da região zigomática, degrau em região infraorbitária, edema e equimose em mucosa jugal, alargamento facial, má oclusão, degrau em pilar zigomático e diplopia ${ }^{1}$.

O diagnóstico de fraturas CZO é geralmente clínico, com confirmação por tomografia computadorizada (TC) $\mathrm{O}$ advento desse exame representa um grande avanço e facilita muito a localização precisa das imagens, uma vez que não há sobreposição de imagens como nas radiografias convencionais; são utilizadas em cortes axiais e coronais ${ }^{1,3}$.

Frequentemente 0 tratamento das fraturas zigomático-orbitárias é cirúrgico a fim de restabelecer a proteção do globo ocular, quando a fratura se associa a este, e devolver os contornos normais e a proeminência da face, devolvendo assim a estética. O zigoma atua também como dissipador e transmissor das forças mastigatórias, logo, faz-se necessária a reabilitação para devolver a parte funcional do sistema estomatognático. Fraturas do corpo do zigoma e arco zigomático podem interferir significantemente na abertura e fechamento bucal e ainda estar relacionado com parestesia do nervo infraorbital, enoftalmia, diplopia, e limitação na motilidade ocular, o que configura o tratamento cirúrgico como o tratamento de eleição para as fraturas mencionadas ${ }^{5,6}$.

Esse trabalho tem como objetivo elucidar o tratamento de paciente com fratura do complexo zigomático. Além de evidenciar o sucesso do tratamento preconizado ao paciente como retorno a função mastigatória e da sua qualidade de vida.

CASO CLÍNICO

Paciente do sexo masculino, 48 anos, leucoderma, vítima de agressão física foi encaminhado ao Serviço de Cirurgia e
Traumatologia Buco Maxilo Facial do Hospital Universitário Maria Aparecida Pedrossian (HUMAP), para avaliação de provável fratura do complexo zigomático-orbitário esquerdo. Com relação ao histórico médico, o mesmo nega comorbidades sistêmicas e relata cirurgia pregressa de fratura de mandíbula em região de sínfise. Ao exame físico inicial, observou-se equimose periorbitária do lado esquerdo, hiposfagma em globo ocular esquerdo, afundamento em região zigomática e degrau infraorbitário ambos do lado esquerdo, parestesia em lábio superior e asa do nariz a esquerda, alteração em acuidade visual ipsilateral e limitação em abertura bucal (Figuras 1 e 2).

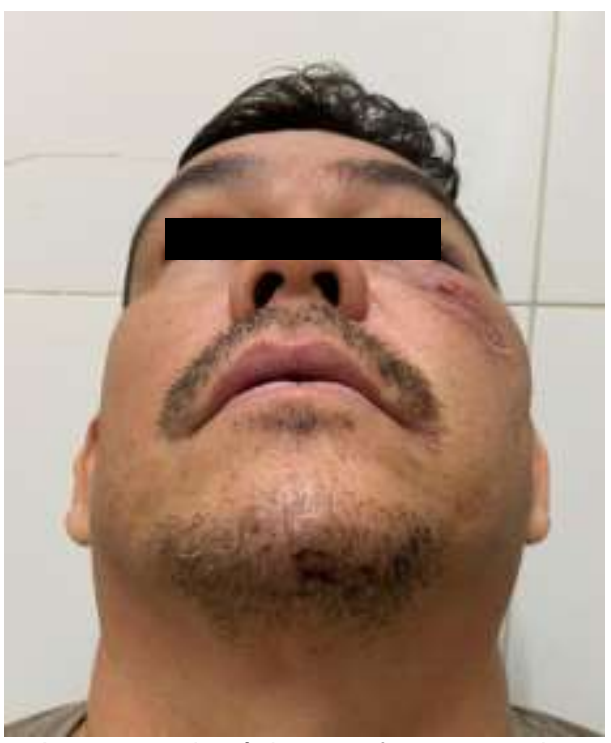

Figura 1: Aumento volumétrico em face esquerda, próximo a região zigomático-orbital.

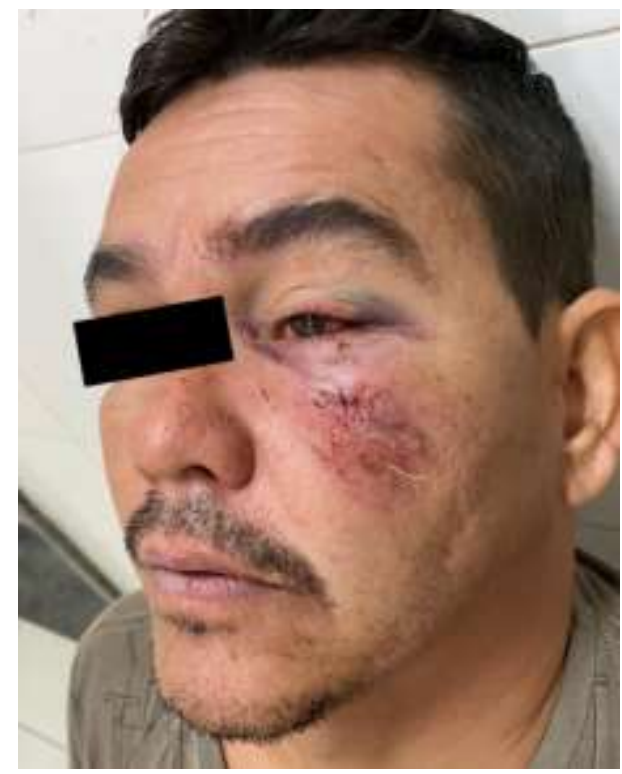

Figura 2: Presença de escoriações, hiposfagma em globo ocular esquerdo e afundamento da região zigomática.

Em tomografia computadorizada de face, nas reconstruções axial, sagital, coronal e 3D (Figura 3) e radiografias póstero-anterior de Water (Figura 4), axial de Hirtz para arco 
zigomático (Figura 5) e póstero-anterior de face (Figura 6), observou-se fratura em complexo zigomático orbitário esquerdo com deslocamento em seus pilares zigomaticomaxilar, frontozigomático, nasomaxilar (pilar canino) e deslocamento em rima infraorbitária ipsilateral. Nota-se material de osteossíntese em região sinfisária. Após avaliação de risco cirúrgico pelo Serviço de Cardiologia, o paciente foi submetido a procedimento cirúrgico sob anestesia geral de redução cruenta e osteossíntese.

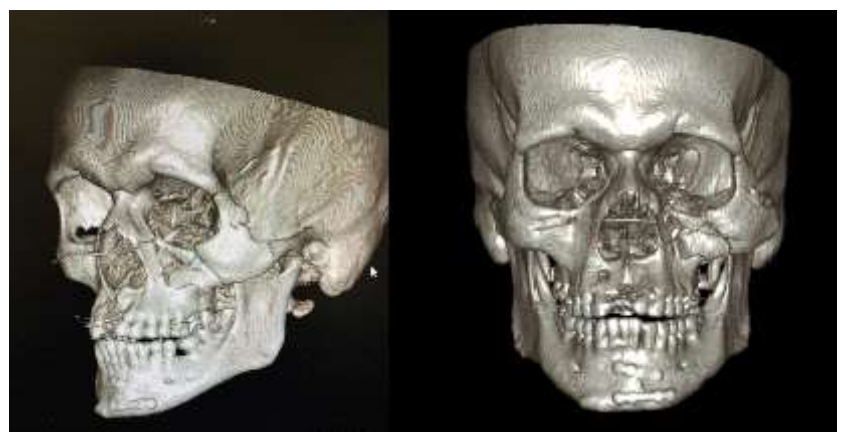

Figura 3: Tomografia computadorizada, reconstruções 3D.

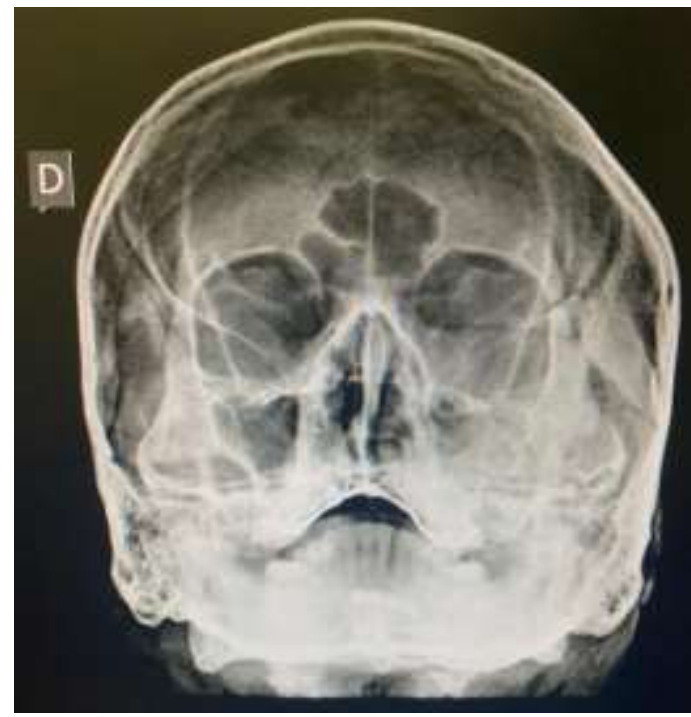

Figura 4: Radiografia póstero-anterior de Waters.

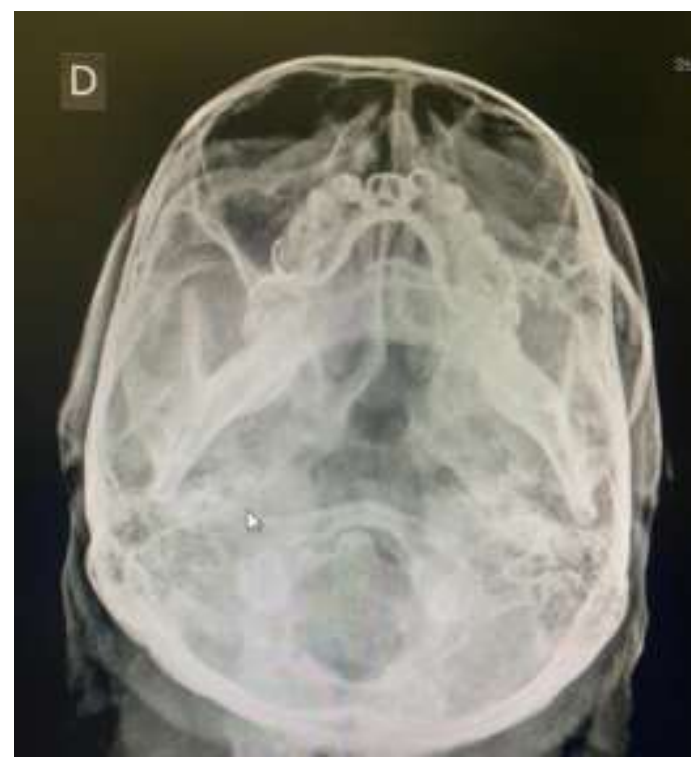

Figura 5: Radiografia axial de Hirtz para arco zigomático.

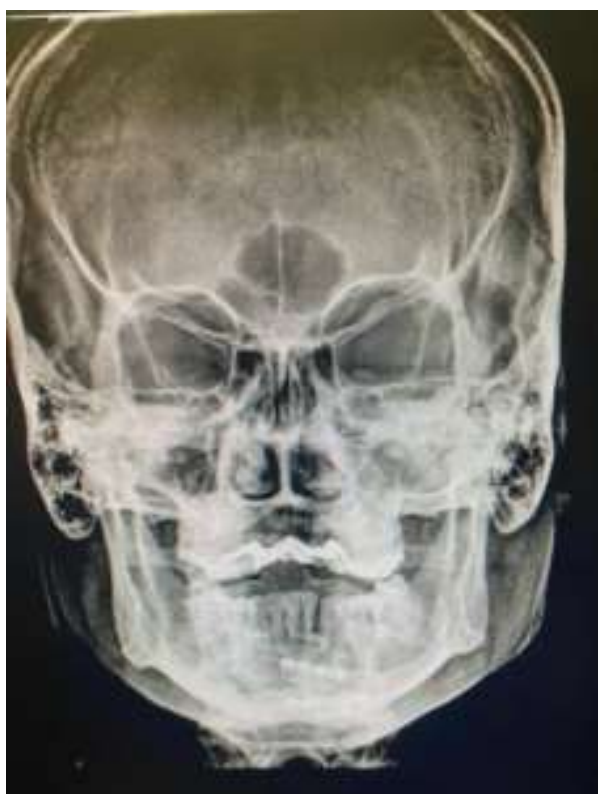

Figura 6: Radiografia póstero-anterior de face.

Foram realizados os acessos subtarsal visando a fratura infraorbitária, o acesso superciliar objetivando a fratura em sutura frontozigomática (Figuras 7 e 8 ) e 0 acesso intraoral (Figura 9) observando as fraturas em pilares zigomaticomaxilar e nasomaxilar. Foi realizada, então, a redução das fraturas e fixação com placas e parafusos de titânio do sistema 1.5 do pilar frontozigomático, rima infraorbitária, pilar zigomaticomaxilar e nasomaxilar (Figura 10), respectivamente.

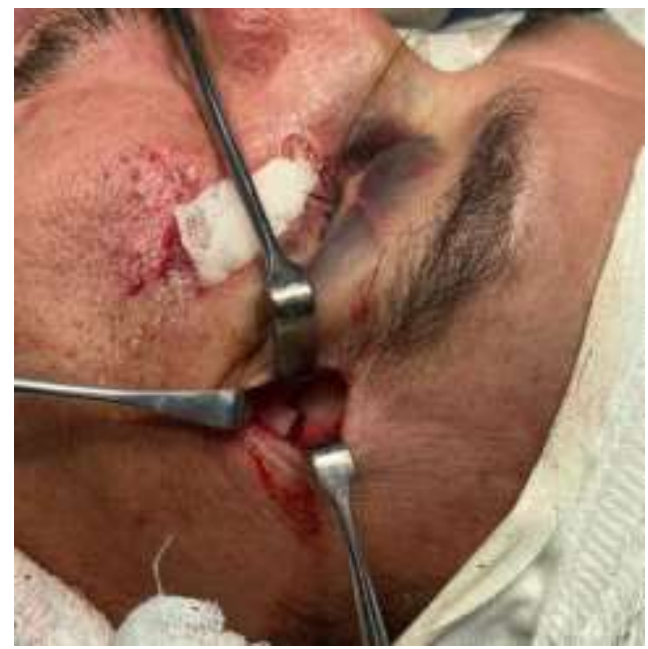

Figura 7: Acesso superciliar.

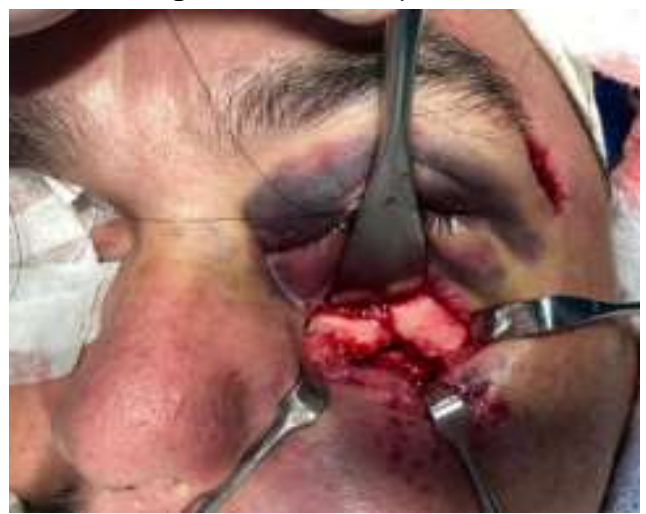

Figura 8: Acesso subtarsal. 


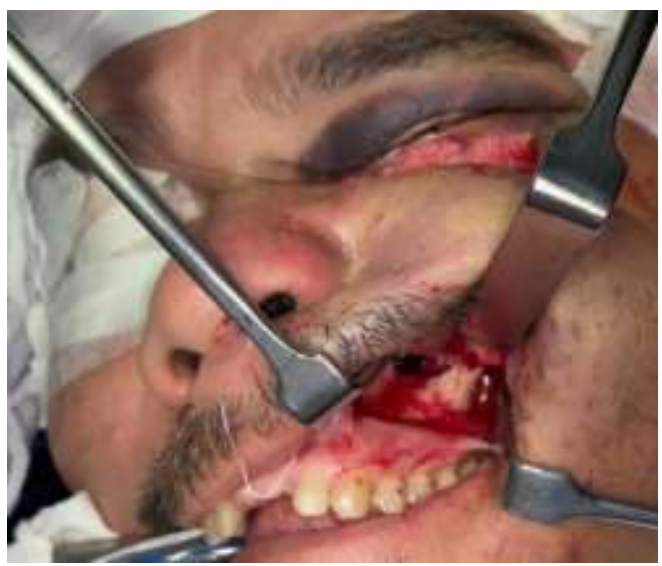

Figura 9: Acesso intraoral.

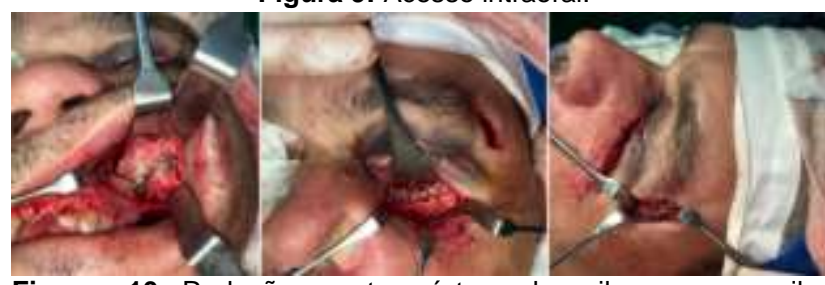

Figuras 10: Redução e osteossíntese dos pilares nasomaxilar, zigomaticomaxilar $(A)$, rima infraorbital $(B)$ e frontozigomático $(C)$.

Após fixação foi notado boa estabilidade do complexo e reestabelecimento da oclusão de forma satisfatória, sendo então, realizado as suturas dos acessos superciliar e subtarsal em seus planos profundos com pontos interrompidos com Vicryl ${ }^{B}$ 4-0 (Ethicon, Johnson \& Johnson Medical Devices, São Paulo - Brasil) e em derme sutura contínua intradérmica com nylon 5-0. No acesso intraoral foi realizada sutura interrompida em planos profundos com Vicryl® 4-0 e em mucosa, foi realizada sutura contínua festonada com Monocryl® 4-0 (Ethicon, Johnson \& Johnson Medical Devices, São Paulo, Brasil).

Ao exame radiográfico pós-operatório, notou-se boa redução das fraturas e sistemas de fixação em posição. Ao exame clínico no primeiro dia de pós-operatório, paciente encontrava-se no leito, em bom estado geral, sem queixas álgicas, sem queixas visuais, apresentando edema em região periorbitária a esquerda compatível com o procedimento, hiposfagma a esquerda, suturas em acessos subtarsal e superciliar em posição e sem secreções. Ao exame intrabucal, paciente apresentou boa oclusão dentária, sutura em acesso maxilar a esquerda em posição e boa higiene oral.

Foi realizada a alta hospitalar do paciente, acompanhada de orientações quanto aos cuidados, prescrição medicamentosa via oral analgésica, anti-inflamatória e antibiótica, prescrito também colírio para lubrificação do globo ocular esquerdo, com retorno programado em ambulatório para acompanhamento.

Ao $28^{\circ}$ dia de pós-operatório, o paciente apresentou ptose palpebral inferior do olho esquerdo, que o mesmo relata que já havia apresentado episódios prévios ao trauma, regressão do hiposfagma, acessos cirúrgicos em boa cicatrização, relatando parestesia e praxia em região zigomática. Além disso, a oclusão dentária encontrava-se estável e satisfatória, foi prescrito colírio lubrificante para evitar danos a conjuntiva do globo ocular esquerdo e realizado encaminhamento ao ambulatório de oftalmologia devido a apresentação de alteração visual em que o paciente referiu após a cirurgia que já apresentava alteração visual previamente à mesma. Também foi prescrito Citoneurin $\AA$ 5000Ul (Merck Brasil, São Paulo - Brasil), 1 drágea a cada 8 horas, durante 15 dias, devido a parestesia. Foi encaminhado ao ambulatório de fisioterapia para reestabelecimento de movimentos de expressão facial da região infraorbitária e praxia do lado esquerdo. O paciente segue em acompanhamento. DISCUSSÃO

O CZO ocupa a terceira posição dentre as fraturas faciais, sendo que os indivíduos mais acometidos se encontram na faixa etária de 21 a 30 anos $^{4}$ e são do sexo masculino ${ }^{4,5,7}$. Com relação à etiologia dos traumas, os acidentes motociclísticos apresentaram a maior frequência, seguidos de agressão física e acidente ciclístico ${ }^{7}$. No caso presente, o paciente apresentava características semelhantes com as descritas em literatura sobre fraturas do complexo zigomático-orbitário.

O tratamento dessas lesões visa à recuperação da função, com o mínimo de sequela na aparência do paciente. Deste modo, as fraturas da região do CZO representam considerável desafio à traumatologia, já que mantém íntima relação com diversas estruturas faciais e, em decorrência de forças severas, podem ocorrer várias sequelas e/ou complicações.

O objetivo do tratamento das fraturas é restaurar a arquitetura do esqueleto facial. Falhas no correto posicionamento do osso fraturado podem resultar em sequelas que são de difícil correção numa segunda cirurgia ${ }^{1,5}$. Dentre elas, pode-se citar: hematosinus e epistaxe, diplopia, pelo edema e hematoma intracavidade orbital; equimose subconjuntival e diminuição da mobilidade ocular ${ }^{8}$. No caso relatado, o paciente apresentou no primeiro dia de pós-operatório edema em região periorbitária compatível com o procedimento cirúrgico realizado, apresentando hiposfagma ou equimose subconjuntival do lado esquerdo, não apresentando nenhum dos sinais citados acima.

A opção de abordar uma fratura do 
zigoma com redução aberta ou fechada ainda é controversa. No caso exposto, o tratamento proposto foi realizado com redução aberta para melhor redução e estabilização da fratura do grupo IV segundo classificação de Knight e North de $1961^{9,10}$. A fixação interna rígida, que consiste em uma fixação através da utilização de placas e parafusos em fraturas do complexo zigomático podem ser feitas em pilar zigomático, sutura frontozigomática, pilares caninos e margem infraorbitária ${ }^{6,10,11}$.

Em relação ao tratamento da fratura do osso zigomático, não há consenso quanto a melhor técnica. $\mathrm{O}$ grau de deslocamento assim como da fragmentação dos cotos fraturados ${ }^{8}$ acrescido da escolha do material para fixação, seja ele rígido ou o uso de fios de aço para esse fim sem esquecer-se da experiência do cirurgião no uso do material enquadram-se como requisitos essenciais para 0 sucesso do tratamento ${ }^{6,8}$.

Alguns autores citam a fixação em três pontos sendo indicada quando a fratura do complexo zigomático se encontra instável. Optamos por realizar a osteossíntese com placas e parafusos de fixação interna rígida seguindo este padrão citado, em três pontos (margem infraorbital, pilar frontozigomático e pilar zigomaticomaxilar) $5,7,12$.

No tratamento cirúrgico alguns acessos foram considerados para os pilares comprometidos (pilar frontozigomático, zigomáticomaxilar, e o horinzontal, moldura infraorbital) ${ }^{13}$. Para região de rebordo infraorbital, o acesso de eleição foi o sub-tarsal, devido aos menores índices de complicações e facilidade de técnica, se comparado ao acesso sub-ciliar ou transconjultival ${ }^{5,6,9}$. A escolha do acesso sub-tarsal, se deu em virtude de seu menor índice de retração palpebral, consequentemente menor exposição da esclera no pós-operatório tardio, e menor índice de ectrópio cicatricial quando comparado ao acesso sub-ciliar ${ }^{2,5}$. No caso relatado, como a paciente apresentava dificuldades na acuidade visual e parestesia em lábio superior e asa do nariz, optou-se por redução da fratura do arco zigomático por via aberta, com a utilização de placas e parafusos do sistema $1,5 \mathrm{~mm}$, devolvendo a estabilidade da região.

Em relação à fratura do corpo do osso zigomático, apenas a fixação por via intrabucal se mostrou satisfatória, devolvendo a estética ao paciente bem como a função, por meio da colocação de uma placa em pilar zigomáticomaxilar e em pilar canino, ambos do lado esquerdo e do sistema $1,5 \mathrm{~mm}$; sendo corroborado pela literatura ${ }^{8}$. Como o degrau palpável em rebordo infraorbitário, a limitação de abertura bucal, 0 déficit visual e 0 afundamento em região zigomática eram os principais sinais apresentados, o caso foi resolvido com sucesso.

O tratamento cirúrgico das fraturas do complexo zigomático e arco zigomático estão indicados quando há presença de fraturas com deslocamentos significativos, associados a limitação da abertura bucal, comprometimento da motilidade e funções oculares, assim como defeitos estéticos na face que geram assimetria facial, como queixa no presente caso ${ }^{6,14,15}$.

Não apenas o tratamento cirúrgico, mas também o acompanhamento clínico é imprescindível para todos os casos, pois suas complicações e sequelas podem gerar déficits sensoriais e, por vezes, funcionais em longo prazo $^{2,10,15}$. O paciente encontra-se com bom aspecto das abordagens cirúrgicas e resultado funcional e estético satisfatórios, porém o paciente não compareceu à outras consultas pós-operatórias.

O bom posicionamento das fraturas abordadas no caso acima mencionado proporcionou uma correta função nos movimentos de abertura e fechamento bucal além da correta projeção ocular e estética facial. Em traumas faciais, o importante a ser ressaltado é a individualização de cada caso, para oferecer o melhor tratamento possível levando em consideração os recursos e tratamentos disponíveis atualmente. Até o momento pode-se considerar sucesso estético e funcional ao caso evidenciado acima ${ }^{2,5,12,15}$. CONSIDERAÇÕES FINAIS

Neste caso, a técnica cirúrgica aberta trouxe um bom resultado, inclusive para maior estabilidade do arcabouço facial comprometido no trauma. O bom posicionamento das fraturas abordadas proporcionou uma correta função dos movimentos de abertura e fechamento bucal, além de correta projeção ocular e estética facial. $O$ tratamento multidisciplinar foi fundamental para a resposta desejada e futura recuperação completa do paciente. Até o momento, pode-se considerar sucesso funcional ao caso apresentado com bons resultados no pós-operatório. Espera-se que o paciente se mantenha com as funções mastigatória e estética preservadas, e com isso se mantenha seu bem estar e qualidade de vida.

\section{REFERÊNCIAS}

1. Paulesini Junior $W$, Farias LP, Aquati $M$, Rapoporat A, Leporace AA. Fratura de Complexo Zigomático: Relato de caso. Rev Odontol Univ São Paulo. 2008;20(3):301-6.

2. Colombo LT, Santos GM, Gonçalves PZ, Fabris 
AL da S, Souza FA, Faverani LP et al. Fratura do complexo zigomaticomaxilar por agressão física: relato de caso. Arch Health Invest. 2017;6(8):390-93.

3. Starch-Jensen T, Linnebjerg LB, Jensen JD. Treatment of Zygomatic Complex Fractures with Surgical Nonsurgical Intervention: A Retrospective Study. Open Dent J. 2018;12:377-87.

4. Zamboni, RA, Wagner JCB, Volkweis, MR, Gerhardt EL, Buchmann EM, Bavaresco CS. Levantamento epidemiológico das fraturas de face do Serviço de Cirurgia e Traumatologia Bucomaxilofacial da Santa Casa de Misericórdia de Porto Alegre - RS. Rev Col Bras Cir. 2017;44(5):491-97.

5. Mendonça JC, Gaetti-Jardim EC, Santos MA, Ximenes WLA, Santos CM, Quadros DC et al. Tratamento cirúrgico de fratura do complexo zigomático orbital: relato de caso. Arch Health Invest. 2016;5(5):251-55.

6. Santos GM, Akabane STF, Reis EM, Pires WR, Coléte JZ, Souza FA, Faverani LP et al. Fratura de complexo zigomaticomaxilar por prática esportiva: relato de caso. Arch Health Invest. 2017;6(4):150-54.

7. Lee El, Mohan K, Koshy JC, Hollier LH Jr. Optimizing the surgical management of zygomaticomaxillary complex fractures. Semin Plast Surg. 2010;24(4):389-97.

8. Obuekwe O, Owotade F, Osayuwu O. Etiology and pattern of zygomatic complex fractures: a retrospective study. J Natl Med Assoc. 2005;97(7):992-96.

9. Ellis E III, Zide M. Acessos cirúrgicos ao esqueleto facial. 2. ed. São Paulo: Santos; 2006.

10. Miloro M, Ghali GE, Larsen PE, Waite PD. Princípios de Cirurgia Bucomaxilofacial de Peterson. 3. ed. São Paulo: Santos. 2016.

11. Hupp JR, Ellis E III, Tucker MR. Cirurgia Oral e Maxilofacial Contemporânea. 6. ed. Rio de Janeiro: Elsevier; 2015.

12. Kelley P, Hopper R, Gruss J. Evaluation and treatment of zygomatic fractures. Plast Reconstr Surg. 2007;120 (7 Suppl 2):5S-15.

13. Madeira MC. Anatomia da Face. 8. ed. São Paulo: Sarvier; 2012.

14. Ellis E III, Kittidumkerng W. Analysis of treatment for isolated zygomatic maxillary complex fractures. J Oral Maxillofac Surg. 1996;54:386-400.

15. Gaetti Jardim EC, Santiago Junior JF, Melo RL de, Mendonça JCG de, Faverani LP, Garcia Junior IR et al. Combinação de Técnicas para Tratamento Cirúrgico de Fratura do Complexo Zigomático-Maxilar: Relato de Caso. Arch Health Invest. 2013; 2(3):33-6.

\section{CONFLITO DE INTERESSES}

Os autores declaram não haver conflitos de interesse AUTOR PARA CORRESPONDÊNCIA

\section{Gaya Bertoldo Cassiano}

Avenida Noroest, 1583. Amambaí

79009-760. Campo Grande - MS, Brasil

E-mail: gaya.bertoldo@hotmail.com 